\title{
MENTAL MODEL AND NETWORKS-BASED METHODOLOGIES FOR THE DEVELOPMENT OF AHP/ANP STRUCTURES
}

\begin{abstract}
In this article we propose a methodology based on mental models and network analyses for the identification of criteria and alternatives for AHP modeling.
\end{abstract}

Keywords: mental models, networks, methodologies, stakeholders, water

\section{Introduction}

The process of generating an AHP/ANP models through workshops may be demanding in terms of economic resources, personnel and time. In this paper we present a method for the identification of criteria and alternatives based on mental models (Jones 2011) and network analyses (Fernández et al. 2015). We believe this can be a complementary methodology to the AHP/ANP modeling. This paper is framed in the context of the project MEGADAPT. The methods introduced here uses data from interviews and workshops with stakeholders from the locality of La Magdalena Contreras, Mexico City. This locality has the last remaining free-flowing river within the city. Actors of Magdalena Contreras experience problems of water such as water scarcity and flooding.

\section{Hypotheses/Objectives}

The aim of our work is to generate a methodology based on mental models and networks analysis for the identification of stakeholder's criteria and alternatives as an input for the AHP/ANP model (Eakin \& Bojorquez 2008). We pretend to create a methodology that can be generalized.

\section{Research Design/Methodology}

With an Institutional Analysis and Development (IAD) Framework (Ostrom 2009), we found eleven key actors from the government and civil society. Seven semi-estructured interviews and four participatory workshops were applied in order to elicit a mental model for each actor.

Mental models were systematized in a graph in which ideas and concepts are connected with each other by relations of influence (e.g., water leaks has an influence on water scarcity). In order to compare different mental models, those ideas and concepts contained in them that were similar, were standardized in a single term. Standardized terms were mapped into a binary proximity matrix for each actor's mental model. Every mental model was converted into a network using NetworkX, a Python library for network analysis. Pairs of connected terms were extracted from the intersection of two or more networks, as if they were sets (i.e., Network-A $\cap$ Network-B).

The pairs of connected terms, obtained from the intersection of networks, become part of a new edgelist (a list of pairs of terms) that would be represented as a network. We assume that every pair of connected terms represents a composed idea (a complex idea). Because we extracted pairs of terms from the original mental model matrix, we conserved the direction of the edge. We also gave a weight to every edge by adding-up the times a pair of connected terms has been shared among networks. In this way, we ended-up with terms connected by directed and weighted edges. 
When these methods are applied, the resulting set of networks may be more or less complex, according to the number of nodes and edges. Every network is a composition of shared pairs of connected terms that come from different mental models.

\section{Results}

In order to validate our results, we compared them to an AHP model, previously generated. The AHP model was created by performing workshops with the people from the same community. The resulting network contains six subgraphs. Every graph represents a mixture of criteria and alternatives. The content of these subgraphs had a correspondence with our AHP model. For example, in the AHP from local government workshop we found that they shared "lack of information" and "untrustworthiness" as common criteria, and "institutional resources investment" and "solution of conflicts" as common alternatives.

Another example of AHP comes from the civil society workshop. We found that they share criteria, such as "urban growth" and "settlements" and "urban growth" and "regular and irregular settlements". At the same time, we also found "services management" and "working groups" (with stakeholder and government actors) as common alternatives.

\section{Conclusions}

Next step is to validate the resulting network with the stakeholders so they can generate their own AHP model. This methods rely on formal processes in order to minimize researchers' subjectivity. If we prove this methodology trustworthy, it could be systematized and generalized as an inexpensive way of generating AHP models. Finally, this can be a complementary methodology for other wellestablished methods for AHP modeling that might bring robustness to the model.

\section{Key References}

Jones, N., Ross, H., Lynam, T., Perez, P., \& Leitch, A. (2011). Mental models: an interdisciplinary synthesis of theory and methods. Ecology \& Society, 16(1): 46.

Fernández, N., Carreón, G., Cortés LE., Gershenson, C., \& Siqueiros-García, JM. (2015) Network Analysis and Text Mining Approach for Moorland Systems Management and Self-Governance. (October 6, 2015). Available at SSRN: http://ssrn.com/abstract $=2670019$

Eakin, H., and L. A. Bojórquez-Tapia. (2008). Insights into the composition of household vulnerability from multicriteria decision analysis. Global Environmental Change, 18: 112-127.

Ostrom, E. (2009) A General Framework for Analyzing Sustainability of Social-Ecological Systems. Science 24: 419-422. 